\title{
In-situ stylus profilometer for a high frequency reciprocating tribometer
}

\author{
*T.J. Kamps ${ }^{\text {a }}$, J.C. Walker ${ }^{a}$, A.G. Plint ${ }^{\text {b }}$ \\ aNational Centre for Advanced Tribology at Southampton, Engineering and the Environment, \\ University of Southampton, Highfield, Southampton SO17 1BJ, UK

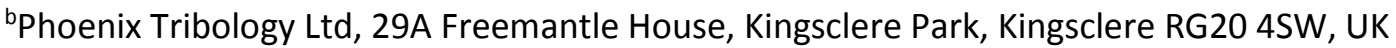 \\ *Corresponding author email address: T.Kamps@soton.ac.uk
}

Keywords: Profilometery, sliding wear, start-stop, cast iron, running-in, wear transitions

\begin{abstract}
Measuring the friction and wear characteristics of a tribological contact is essential to gaining a detailed understanding of its performance and predicted life. Wear rate and friction coefficient measurements are obtained from instrumented benchtop tribometers designed to replicate specific tribological contacts. Due to the difficulty of measuring wear in situ, measurements are typically made before and after an experiment. The wear rate must be assumed to be linear for it to be used to predict product life, however this is assumption can hide changes occurring during an experiment which indicate wear transitions. This paper details the design and validation of an in situ stylus profilometer for a reciprocating sliding tribometer to provide an insight into the wear transitions occurring during dry sliding of 52100 bearing steel against graphitic flake cast iron. The profilometer's performance was validated using ground roughness standards and the accuracy found to be approximately $110 \mathrm{~nm}$. Incubation, run-in and steady state wear regimes were identified by the profilometer and corroborated with friction coefficient data, providing an enhanced understanding of the tribological contact behaviour.
\end{abstract}

\section{Introduction}

Tribology seeks to understand the behaviour of surfaces in relative motion. The relationship between friction and wear is central to the discipline as it defines the efficiency and life time of a contact. These characteristics are influenced by the material and geometry of the surfaces as well as the chemistry of the contact environment. The environmental and financial impact of building and testing a complete complex system equipped with sensors to optimise one component for these parameters can be prohibitive. Benchtop tribometers offer a cost saving alternative, as they use representative surfaces which are usually smaller than the original components and are specifically designed to measure wear and the frictional energy dissipated in the contact.

Wear regime changes may be caused by an external change in contact conditions such as a variation in load [1,2], or by an evolution of the contact conditions caused by steady state friction and wear [3]. Induced changes in wear regimes can sometimes be predicted however changes arising from the contact evolution are often unexpected and consequently much more interesting. For example the 
quasi stability of a cyclic wear mechanism such as the removal and reforming of an oxide film during sliding wear lends itself to using a high resolution in situ wear detection technique [4][5].

During a wear transition, such as scuffing for example, contact surfaces experience alteration in the form of plastic deformation or material removal [6-8]. The wear severity determines whether the contact can return to its previous wear regime or whether it has been significantly modified making the wear transition permanent [9]. In these cases measuring wear during the experiment can be beneficial as a combined knowledge of wear and friction characteristics are often crucial to understanding wear transitions and essential for designing a successful tribological contact.

Wear-in or run-in is a particular type of wear transition caused by contact evolution that occurs early during the contact lifecycle. During this regime, surface asperities are worn away removing fine geometric intolerances to make a conformal contact and at the same time reducing the contact pressure [10-12]. When an equilibrium is established the contact enters a period of steady state or linear wear, Figure 1.

In some cases run-in is proceeded by an incubation period, for example where an oxide film protecting one or both surfaces is gradually worn away, or where material is initially transferred between sliding surfaces before being removed from the contact [13], Figure 1.

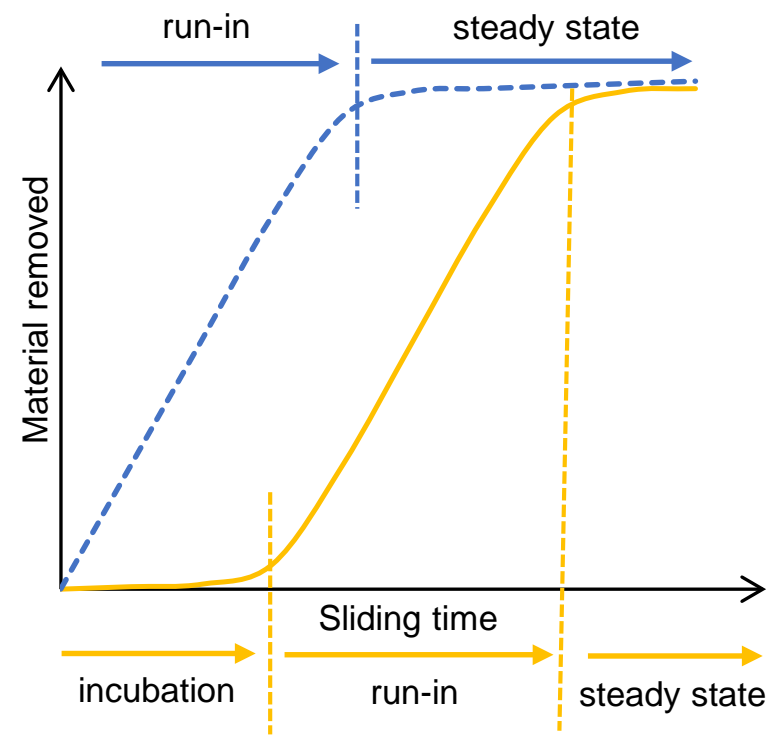

Figure 1: Two example sliding wear behaviours: run-in followed by steady state (blue) and incubation followed by run-in and steady state (yellow), adapted from [13].

The performance of a tribological contact is intrinsically related to the topography of the surfaces. A large group of functional parameters (e.g. roughness, skew, kurtosis), can be determined from profile measurements to numerically describe the topographical properties of a surface. These can be used to ensure satisfactory performance when designing tribological contacts, for example bearing area curves are used to determine load support and lubricant retention properties of plateau honed cylinder liners [14]. They may also be used to assess the severity and type of wear that has occurred on the surface of a worn component.

It is common practice to characterise a surface before and after an experiment and assume that the measured wear occurred at a constant rate throughout the experiment $[15,16]$. Whilst wear rates in specific regimes are often linear and make predicting component life straight forward using this 
method, changes in contact conditions can influence the energy dissipated in the contact and may cause a transition to another wear regime. The ability to monitor wear and surface topography during an experiment allows wear mechanisms and transitions (e.g. incubation, run-in and steady state) to be identified. This is important as it allows the wear rates to be separated and consequently a more accurate prediction of component life can be achieved [17].

However there exist limitations to widespread implementation of continuous wear monitoring during such experiments. As mentioned above, tribometers often use test surfaces smaller than the original part to reduce cost, consequently the contact geometry can result in an increased overlap parameter, the ratio of the contact area to the wear track [18]. When tribometers with reciprocating contacts operate with short stroke lengths and consequently large overlap parameters, the wear track is never sufficiently exposed by the counter surface for measurement. This presents a challenge for directly observing and measuring wear of a surface during an experiment by optical techniques such as laser reflectance or interferometry [19].

Other techniques measure the average displacement resulting from wear across the entire contact area of both surfaces continuously during an experiment and consequently do not require the wear track to be exposed. Examples include the use of linearly variable differential transformers to measure the displacement of a pin in a pin on disk machine $[19,20]$ or capacitance probes to measure the vertical displacement of the reciprocating assembly in a reciprocating tribometer [21]. However in order to separate the wear occurring to both surfaces another complimentary technique is required such as ultrasound, which has been used to measure the wear occurring exclusively to a pin in a pin on disk tribometer [22]. All the described techniques are limited to severe wear measurements as the spatial resolution is dictated by the contact area.

Radio nuclide testing is a further technique which enables wear measurements when the wear track is concealed. It is very useful for remotely detecting wear in assembly tests, however it requires calibration against a profilometry technique and necessitates the contact to be lubricated in order to flush the wear debris from the contact and through a detector [23]. Due consideration also has to be given to the health and safety requirements of handling and disposing of activated materials.

Higher resolution wear measurements can be achieved by periodically interrupting an experiment, removing the coupons and examining them using conventional profilometers [24,25]. However this is generally considered an unsatisfactory solution as it relies on the tribometer operator reassembling the parts in exactly the same position, which is time consuming and subject to considerable random error. Incorrect assembly can result in a wear transition as the contact is forced to wear in again.

One approach to addressing some of the issues outlined above is the development of a tactile profilometer synchronised to the tribometer test equipment with the aim of providing periodic high resolution in situ wear measurements without disturbing the contact. A tactile measurement system enables direct measurement of the surface without being limited by the reflective properties of the surface which can inhibit optical measurement methods. The availability of standard and replacement stylus parts also makes a tactile solution economical to implement. This paper outlines the design rationale for developing an in situ profilometer capable of operating autonomously when integrated into a linear reciprocating tribometer. The calibration, assessment of its performance with known roughness standards and finally its implementation to study the running-in wear behaviour of a dry reciprocating sliding cast iron - steel line contact will be discussed. 


\section{Experimental}

This work aimed to construct a robust and cost effective in situ profilometer integrated into a linear reciprocating tribometer. Stylus profilometry was selected as an appropriately resilient technique for in situ measurement as it was capable of measuring surface profiles in the presence of wear debris. The stylus movement was measured using a Hall effect sensor which was investigated for its potential to provide a cost effective measurement of vertical displacement.

\subsection{Profilometer design.}

The in situ profilometer consisted of two parts, the stylus depth measurement assembly and the traversing mechanism, Figures $2 \& 3$. The traversing mechanism comprised of a linear dove tail slide secured in a vertical orientation to the tribometer, with another slide orthogonally mounted to the moving carriage to provide horizontal motion. The stylus depth measurement assembly was attached to the moving carriage of the horizontal slide, enabling it to be moved horizontally and vertically. The slides were actuated by 2-phase bi-polar stepper motors and feedback from magnetic linear encoders of resolution $1 \mu \mathrm{m}$ was used to control position and velocity. The motor drives (ARCUS technology ACE-SDX) were coordinated by a synchronous motion controller (ARCUS technology PMX 2EX), which communicated with the tribometer control system using a digital interface. This enabled automated drive and surface measurements to be carried out as part of an interrupted velocity experiment, as detailed in section 2.3 .

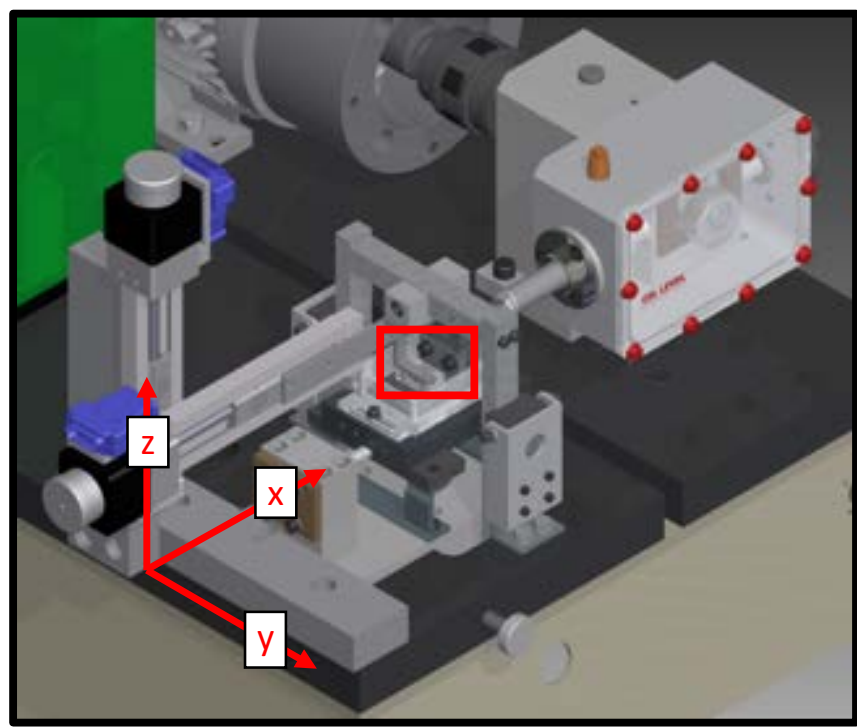

Figure 2: In situ profilometer attached to a TE-77 high frequency reciprocating tribometer.

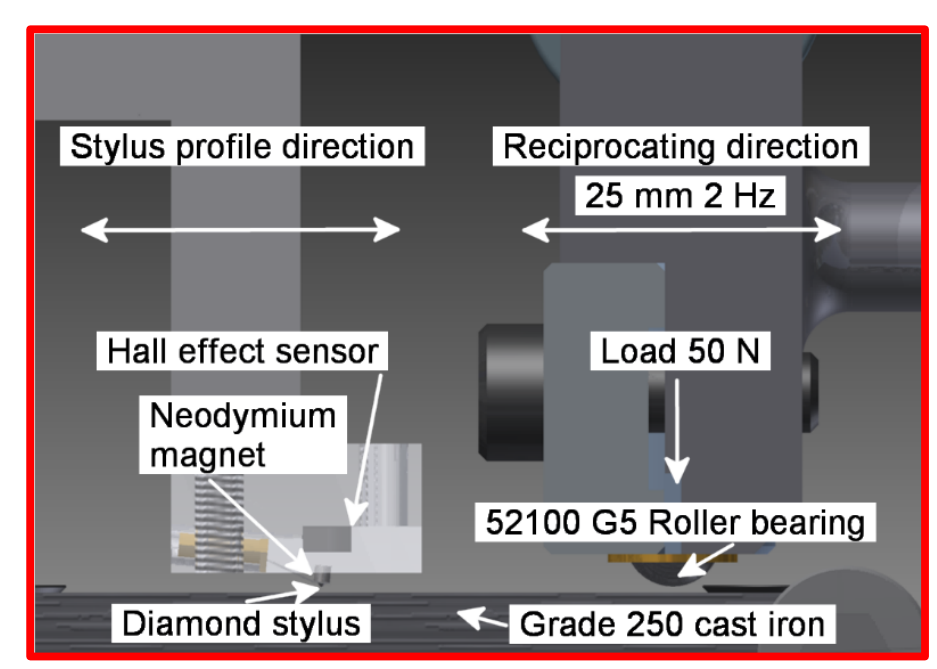

Figure 3: Cross section of the tribometer configuration for line contact dry sliding with the stylus measurement assembly in the measurement position.

The stylus from a Shure N97XE was used as the tactile probe, with an elliptical diamond tip profile, $10 \mu \mathrm{m}$ minor axis and $18 \mu \mathrm{m}$ major axis, as shown in the SEM image in Figure 4. The stylus was oriented such that the major axis was perpendicular to profile direction ( $x$ axis), Figure 2 . The stylus was fixed to a cantilever supported with a rubber bush, which permitted the stylus to move vertically and horizontally. The applied tracking force of the stylus was specified to be in the range $7.4-14.7 \mathrm{mN}$. 


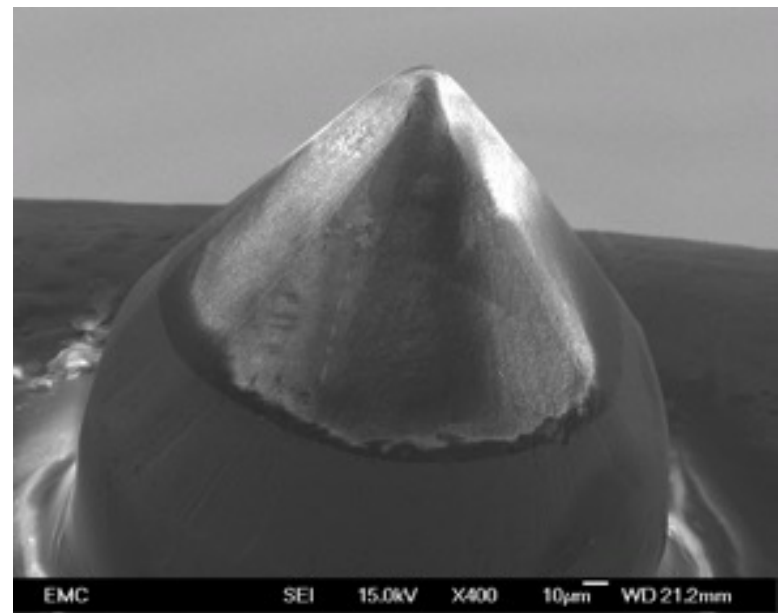

(a)

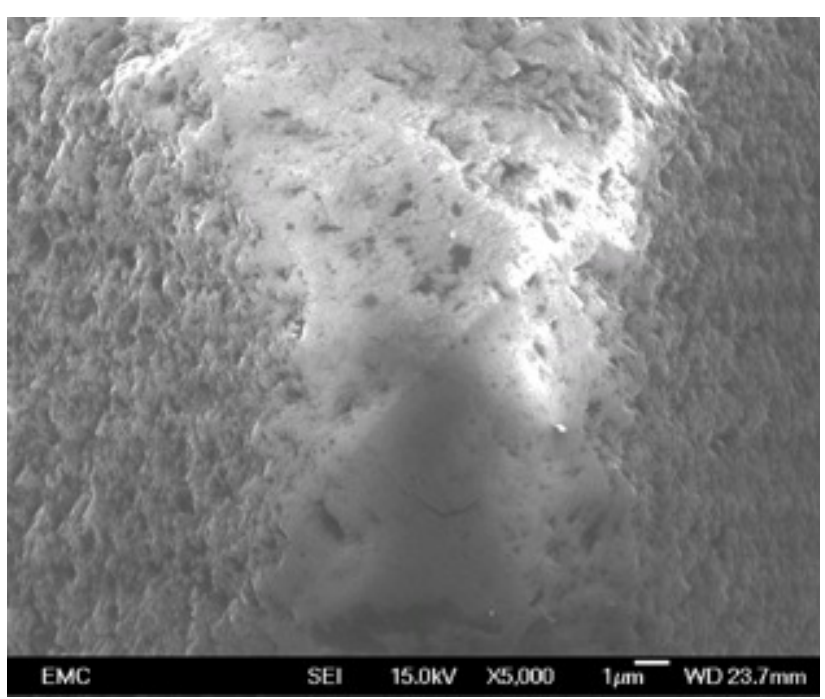

(b)

Figure 4: Secondary electron images of the stylus: (a) Tilted low magnification image and (b) higher magnification image at normal incidence to the tip.

A $1 \mathrm{~mm}$ diameter and $1 \mathrm{~mm}$ length, $(12100 \mathrm{G})$ neodymium magnet was secured to the rear face of the stylus using a cyanoacrylate gel adhesive (Loctite 454$)$. The rubber suspension housing was secured in a polyether ether ketone (PEEK) housing which also contained a ratio metric Hall effect sensor and integrated amplifier (Honeywell SS495A), Figure 3.

From Equation 1, it is possible to calculate that the sensor would be saturated when it was separated from the magnet by less than $0.7 \mathrm{~mm}$. It could also be seen that the magnetic field was negligible at a separation distance of $2 \mathrm{~mm}$, this provided a working distance of approximately $1.2 \mathrm{~mm}$, Figure 5 .

$$
B=\frac{B r}{2}\left(\left[\frac{D+z}{\sqrt{R^{2}+(D+z)^{2}}}\right]-\frac{z}{\sqrt{R^{2}+z^{2}}}\right)
$$

Equation 1

Where: $B=$ magnet field (Gauss), $B r=$ residual flux density (Gauss), $D=$ magnet depth (mm),

$R=$ magent radius $(\mathrm{mm}), z=$ axial displacement from magnet $(\mathrm{mm})$. 


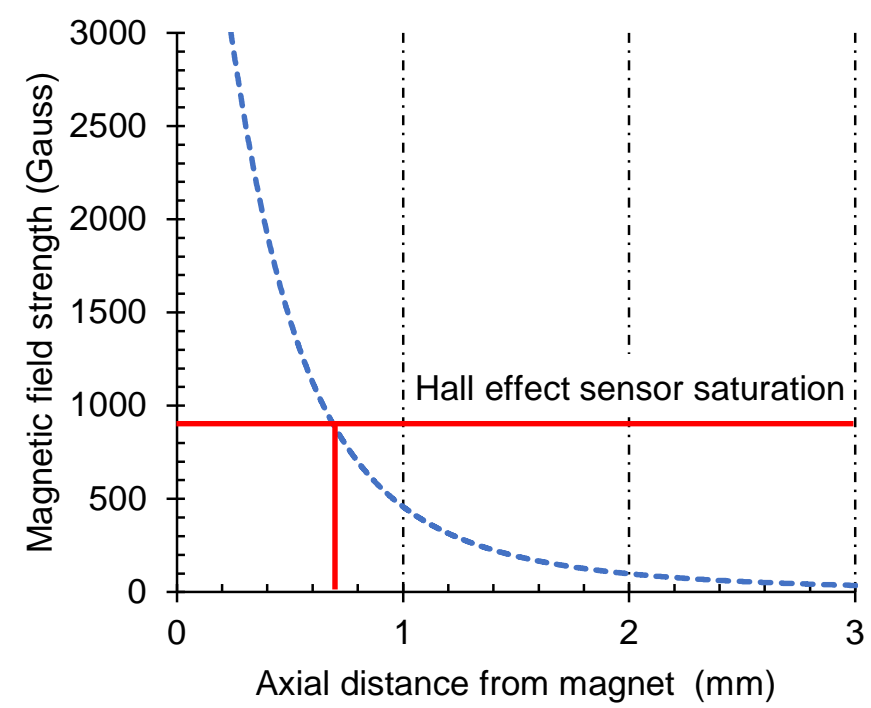

Figure 5: A graph of magnetic field strength against axial magnet displacement.

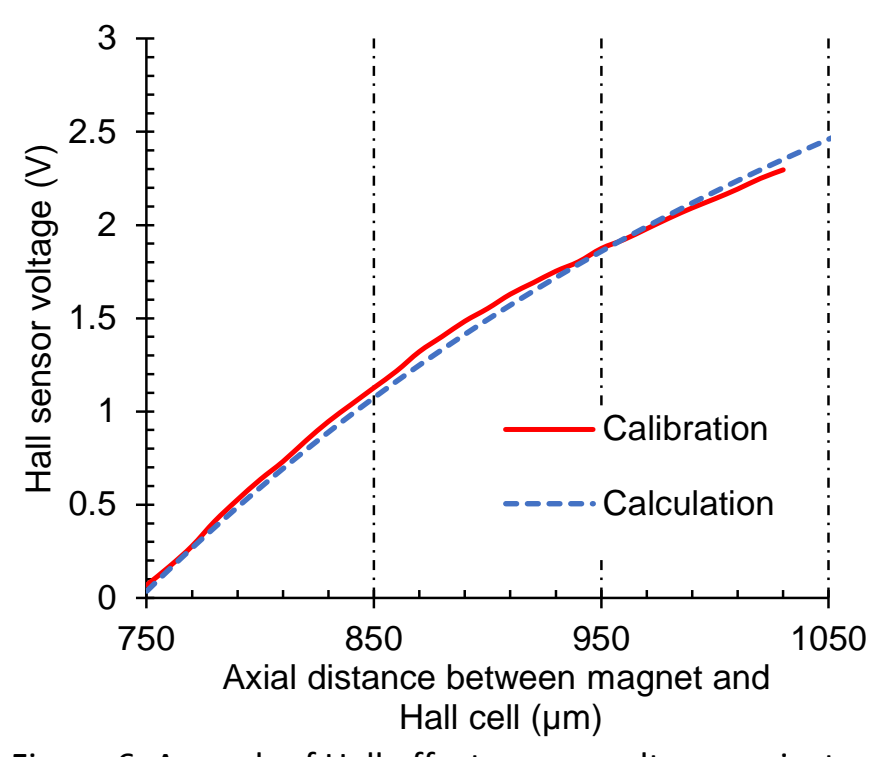

Figure 6: A graph of Hall effect sensor voltage against axial distance between magnet and Hall cell.

The Hall effect sensor provided an output voltage which was linearly proportional to the magnetic flux from the magnet. This was not linearly proportional to the separation distance as the magnetic field was defined by an inverse square relationship, Equation 1 . This had the consequence that the vertical measurement resolution varied according to the separation distance. The sensor had a sensitivity of $0.634 \mathrm{mV} / \mathrm{G} / \mathrm{V}$ at the maximum permitted supply voltage $(10 \mathrm{~V})$ which provided a resolution of $6.34 \mathrm{mV} / \mathrm{G}$. A micrometer was used to calibrate the sensor by varying the axial displacement of the magnet from the sensor, which behaved linearly above $930 \mu \mathrm{m}$ displacement with a gradient of $10 \mathrm{mV} / \mu \mathrm{m}$, Figure 6 .

As the stylus assembly traversed a flat surface at a velocity of $0.5 \mathrm{~mm} / \mathrm{s}$, the needle moved in the vertical ( $z$ axis) varying the magnetic flux passing through the sensor. The vertical depth measurement was discretised at $1 \mathrm{kHz}$ using a high-speed data analogue to digital converter (Data translation DT300). This gave a lateral resolution of $0.5 \mu \mathrm{m}$, which was smaller than the minor axis of the stylus tip. The analogue to digital converter (ADC) had a $0.3 \mathrm{mV}$ resolution, which permitted a maximum possible vertical resolution of $0.03 \mu \mathrm{m}$. The Hall Effect sensor was calibrated with the stylus depth measurement assembly using the vertical encoder (accuracy of $0.04 \mu \mathrm{m} / \mathrm{mm}$ ) attached to the side of the linear dovetail slides. A quadratic equation was fitted to the calibration data to calculate the stylus displacement as a function of the Hall Effect sensor output voltage. The stylus vertical data was transformed from the time to space domain by correlating the velocity of the stylus measurement assembly and the acquisition speed. A total of three measurements were conducted in order to calculate mean and standard deviation values.

\subsection{Roughness standards}

The vertical and lateral performance (precision and accuracy) of the profilometer was evaluated by measuring three surface ground roughness standards manufactured to ANSI B46.1: 1) $0.050 \pm 0.005$ $\mu \mathrm{m}$; 2) $0.40 \pm 0.04 \mu \mathrm{m}$ and 3) $1.60 \pm 0.16 \mu \mathrm{m}$ (Flexbar machine corporation). The profiles obtained were processed by a commercially available software package (Ultra) to calculate the surface roughness using a Gaussian filter according to ISO 4288-1996. A data evaluation length of $0.75 \mathrm{~mm}$ and $\lambda_{C}=0.25 \mathrm{~mm}$ was selected for the $0.05 \mu \mathrm{m}$ roughness standards. The profiles for the $0.4 \mu \mathrm{m}$ and 
$1.6 \mu \mathrm{m}$ standards had an evaluation length of $1.7 \mathrm{~mm}$ and $\lambda_{C}=0.8 \mathrm{~mm}$. A band pass filter of 100:1 was used for all three standards.

\subsection{Dry sliding experiment.}

The in-situ profilometer was used to measure running-in wear during an unlubricated reciprocating line contact experiment. A 52100 roller bearing ( $6 \mathrm{~mm}$ diameter, $10 \mathrm{~mm}$ length) was reciprocated against a flat plate of Grade 250 cast iron (ground at $45^{\circ}$ to the reciprocating direction), at a sliding frequency of $2 \mathrm{~Hz}$, through a stroke of $25 \mathrm{~mm}$ using a high frequency reciprocating tribometer (TE-77 Phoenix Tribology Ltd.), Figure 3. The contact supported a load of $50 \mathrm{~N}$ ( $226 \mathrm{MPa}$ ) at $23{ }^{\circ} \mathrm{C}$ and $55 \%$ humidity. The test sequence consisted of 60 seconds of sliding followed by a surface profile measurement, with the same cycle then repeated 9 times, Figure 7.

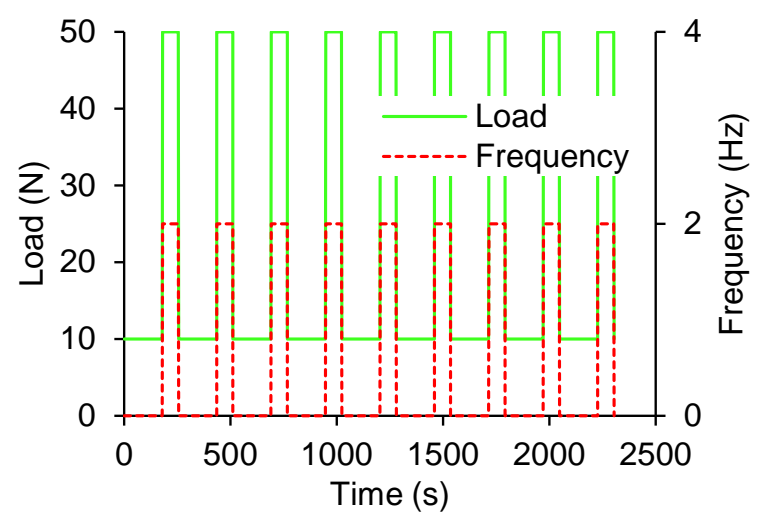

Figure 7: Schematic of test procedure.

Before the experiment, the stylus control software was provided with the initial surface height of the cast iron flat plate measured by the linear encoder, by manually bringing the stylus into contact with the surface, using the Hall sensor for position feedback. This provided the datum for the start of the profile measurement which allowed the stylus to move at high speed from its resting position outside the contact.

After the first minute of sliding, the tribometer's reciprocating arm moved to the furthest end of the stroke making the wear scar accessible for measurement. The stroke position was controlled by a DC motor driving a scotch yoke mechanism with position feedback provided by a linearly variable differential transformer. Due to the torque characteristics of the system at low speed, the contact load was reduced to $10 \mathrm{~N}$ so that the reciprocating arm could be reliably positioned within $2 \mathrm{~mm}$ of the end of the stroke. This exposed half of the wear scar, however only $7 \mathrm{~mm}$ could be measured due to the interference between the clamp on the reciprocating arm and the front of the stylus measurement assembly, Figure 3.

Compressed air was used to remove debris from the surface of wear scar before measurement, then the stylus depth measurement assembly was moved to the start of the profile. The stylus was moved to a height of $1 \mathrm{~mm}$ above the surface using the vertical linear encoder. It was then lowered into contact with the surface using feedback from the Hall effect sensor. Once in contact the stylus was lowered further until the sensor measured a magnetic flux in the middle of its range. This permitted a symmetric maximum deviation of $0.5 \mathrm{~mm}$ from the initial surface height to be measured. Consequently, a worn surface lower than the initial height could be measured but also any higher surface formed by the agglomeration of wear debris could be also measured by the stylus. After the surface profile was measured, the stylus was moved clear from the tribometer and experimental cycle continued. 
After 9 cycles the experiment terminated and the cast iron flat plate was removed from the tribometer. The wear scar profile was measured using a Talysurf stylus profilometer equipped with a $2 \mu \mathrm{m}$ stylus, which had a lateral resolution of $0.25 \mu \mathrm{m}$. The profiles from both profilometers were manually levelled and aligned with each other.

\section{Results and Discussion}

\subsection{Profilometer characterisation}

The performance of the stylus was evaluated by two approaches: 1) The resolution and accuracy of the stylus depth measurement assembly; 2) The resolution and accuracy of the traversing mechanism.

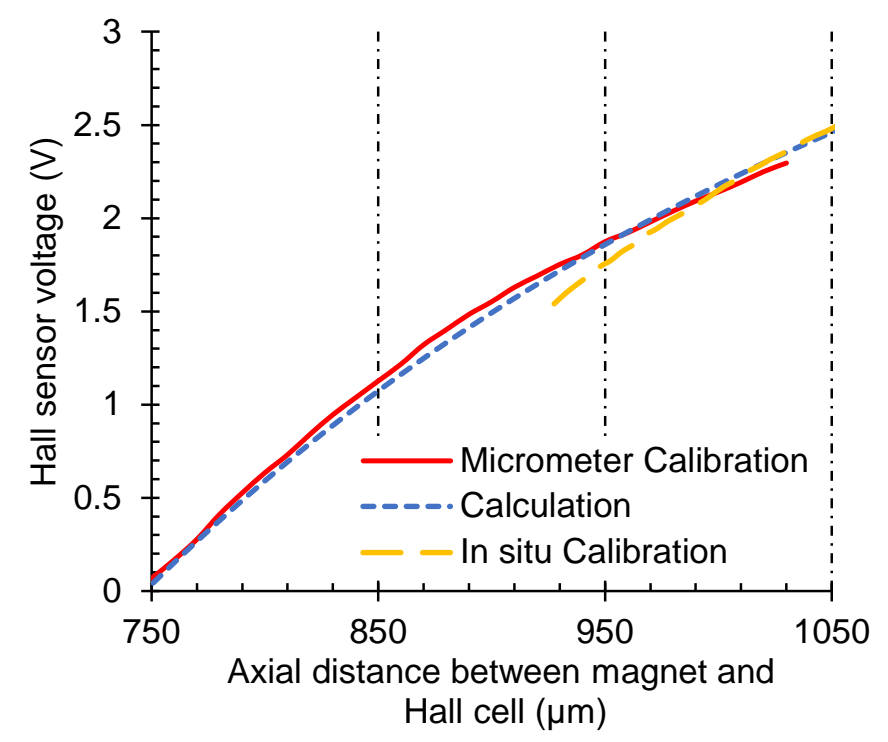

Figure 8: In situ calibration of the Hall effect sensor.

As shown in Figure 8, there was a deviation in the stylus performance at its lower operating range compared to both the predicted and the parallel calibration conducted with a micrometer. The difference in performance between the calibrated Hall Effect voltage and the calculated value was likely to be due to axial misalignment between the magnet and the sensor. The misalignment angle changed as the stylus rotated about its mounting to accommodate change in surface height. The discrepancy between the calculated parallel flux and actual measured magnetic field over this range did not affect the surface height measurement, as a quadratic function fitted to the in situ calibration was used to determine the change in height.

The horizontal resolution was determined by the sampling frequency of the $A D C$, whilst the position resolution was limited by the linear encoders. These provided a resolution of $1 \mu \mathrm{m}$ which was used for closed loop velocity control. The Hall Effect sensor could then be sampled at an independent frequency to provide a suitable lateral resolution, in this case $0.5 \mu \mathrm{m}$.

The vertical resolution achieved by the stylus depth measurement assembly was limited by the presence of electrical noise rather than the resolution of the sensor of the ADC, calculated as 0.03 $\mu \mathrm{m}$. The standard deviation of the total raw data profile when the stylus was stationary was \pm 0.11 $\mu \mathrm{m}$, which provided useful information about the form of wear scars but not sufficient resolution to discriminate between the surface roughness standards detailed in the methodology without postprocessing. In an attempt to reduce the electrical noise content of the signal and increase the 
vertical resolution, a morphological closing filter $[26,27]$ filter was applied to the measured profiles as if they had been traversed by a circle of $10 \mu \mathrm{m}$ in diameter, the measured stylus radius. This removed high frequency components of the signal which were not possible to have measured because of the stylus tip radius. A comparison between the unfiltered and filtered vertical displacement measurements recorded for $0.6 \mathrm{~s}$ while the stylus was stationary is shown in Figure 9. This increased the accuracy of the total vertical measurement to a standard deviation of $\pm 0.07 \mu \mathrm{m}$, at the expense of the horizontal resolution. However the recorded profile was oversampled as the width of the stylus was an order of magnitude larger than the horizontal resolution.

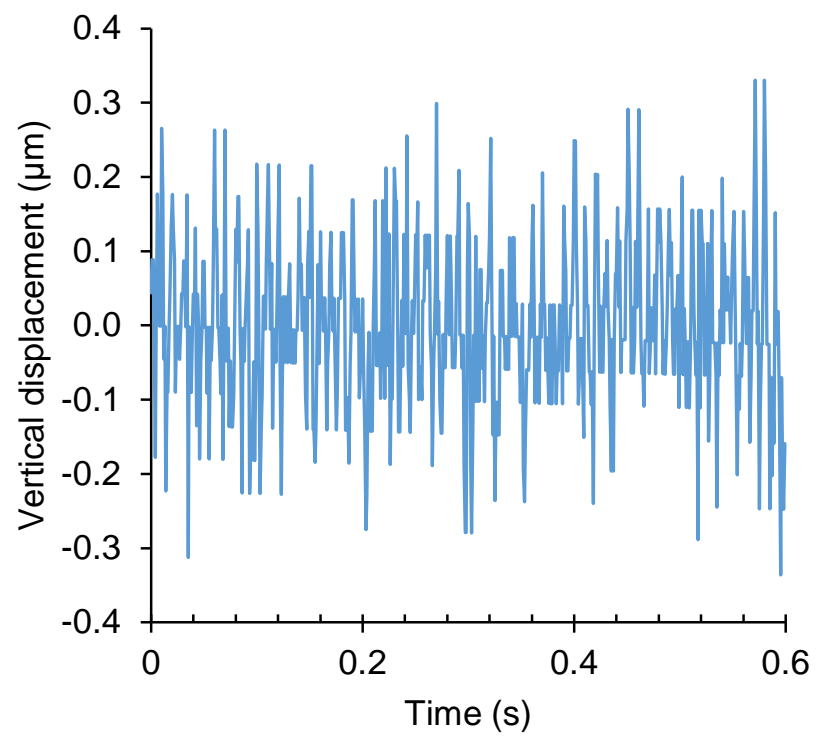

Figure 9: a) Unfiltered Hall effect sensor noise.

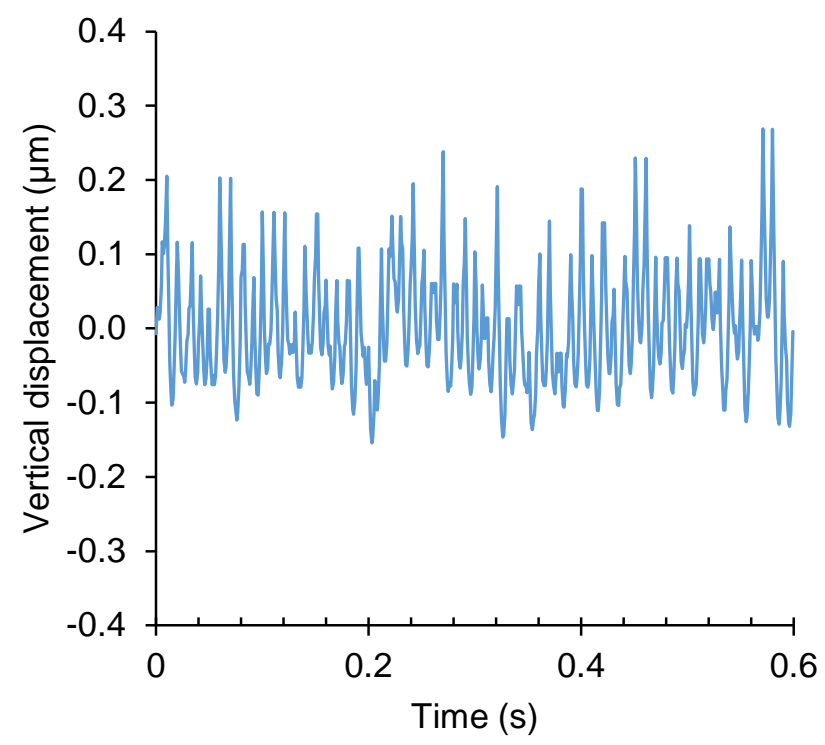

b) $10 \mu \mathrm{m}$ filter Hall effect sensor noise.

\subsection{Evaluation of surface roughness measurements}

Using the morphological closing filter, the in situ profilometer was capable to providing differentiation between the three measured roughness standards with varying degree of accuracy and precision, Figure 10 and Table 1 . The $R_{a}=1.60 \pm 0.16 \mu \mathrm{m}$ standard was measured to be $1.51 \pm$ $0.19 \mu \mathrm{m}$. The $R_{a}=0.40 \pm 0.04 \mu \mathrm{m}$ standard was measured to be $0.52 \pm 0.04 \mu \mathrm{m}$. The $R_{a}=0.05 \pm 0.005$ $\mu \mathrm{m}$ standard was measured to be $0.07 \pm 0.01 \mu \mathrm{m}$.

A possible explanation for the difference between the calibrated and measured roughness was that the in situ stylus velocity was of sufficient magnitude to cause the stylus to separate from the surface during the measurement. This is thought be unlikely as the stylus flexure was designed to operate at a sliding speed of $500 \mathrm{~mm} / \mathrm{s}$ which is three orders of magnitude faster than $0.5 \mathrm{~mm} / \mathrm{s}$ used in this application.

It is more probable that the effect of stylus tip radius was responsible for the discrepancy between the roughness standards and the in situ measurements. The actual surface roughness will have been modified by the stylus geometry during the measurement process [28]. Whether the measured profile is rougher or smoother than the actual profile depends on the radius of the stylus tip in relation to the width of the peaks and valleys on the surface [29]. 


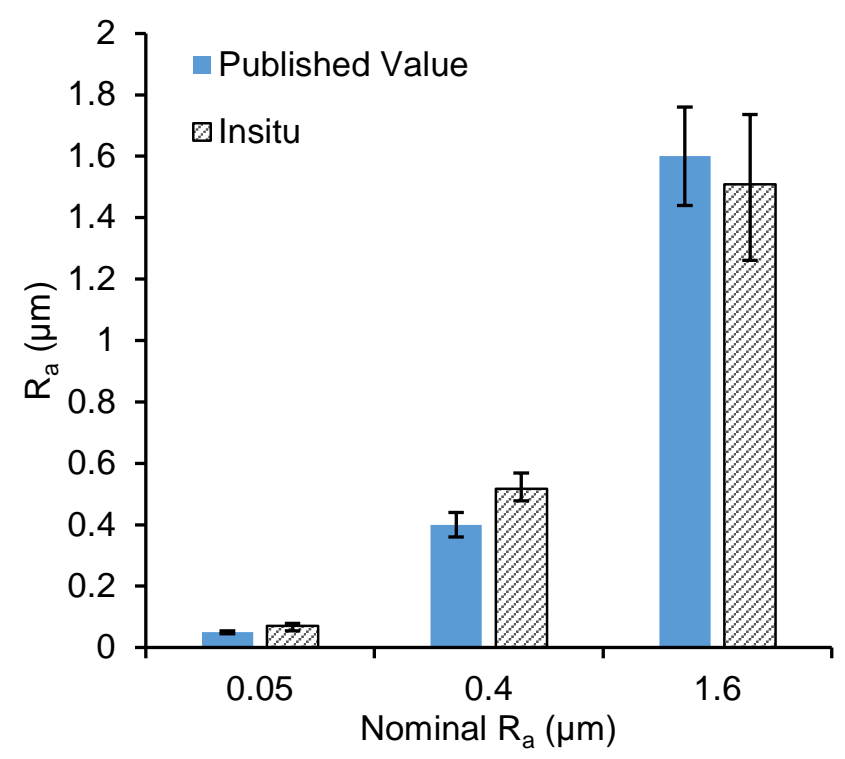

Figure 10: Comparison of in situ stylus and talysurf roughness standards, also include table of this date including data sample length and also cut off frequencies.

\begin{tabular}{r|ccc} 
& \multicolumn{4}{c}{ In situ $(10 \mu \mathrm{m}, \mathrm{x}$ axis $)$} \\
Roughness standard $(\mu \mathrm{m})$ & $\mathbf{0 . 0 5}$ & $\mathbf{0 . 4 0}$ & $\mathbf{1 . 6 0}$ \\
\cline { 2 - 4 }$\lambda \mathrm{C}(\mathrm{mm})$ & 0.25 & 0.80 & 0.80 \\
Sample length $(\mathrm{mm})$ & 0.75 & 1.70 & 1.70 \\
Average Roughness, $\mathrm{R}_{\mathrm{a}}(\mu \mathrm{m})$ & 0.07 & 0.52 & 1.51 \\
Standard Deviation $(\mu \mathrm{m})$ & 0.01 & 0.04 & 0.19 \\
Difference from standard $(\mu \mathrm{m})$ & 0.02 & 0.12 & 0.09
\end{tabular}

Table 1: Measured surface roughness and parameters.

The high magnification SEM image of the stylus tip in Figure 4 suggested that the central apex of the tip could be smaller than the manufacturers published radius $10 \mu \mathrm{m}$, although it is difficult to accurately quantify this from a normal incidence image. The interaction of the stylus flanks with the surface asperities will change depending on the kurtosis of the surface being measured. When the stylus is measuring positively skewed surfaces, where the tip radius is smaller than the surface features, the measured roughness will increase with the tip radius because the true surface is dilated in proportion to tip radius (increasing the measured peak widths and decreasing the valley widths) $[27,28]$.

Conversely if the tip radius is larger than the valley width, which often the case when measuring a rough surface, increasing the tip radius will limit the vertical and lateral resolution. Consequently the measured roughness will be less than the actual roughness as the stylus cannot touch the bottom of the valley in this situation.

This corresponds to the behaviour of the in situ measured roughness compared to the roughness standard values calibrated to ANSI B43.1. The roughest surface was measured to be smoother than its calibrated value, and the smoother roughness were measured to be rougher than the calibrated values. In the case of the smoothest sample, $R_{a} 0.05 \mu \mathrm{m}$, the roughness was below the resolution of the linear operation of the sensor. Whilst the sensor operates at a higher resolution over part of its 
range, the measured roughness for this sample was equal to the roughness of noise recorded when the stylus was stationary.

\subsection{Dry sliding experiment}

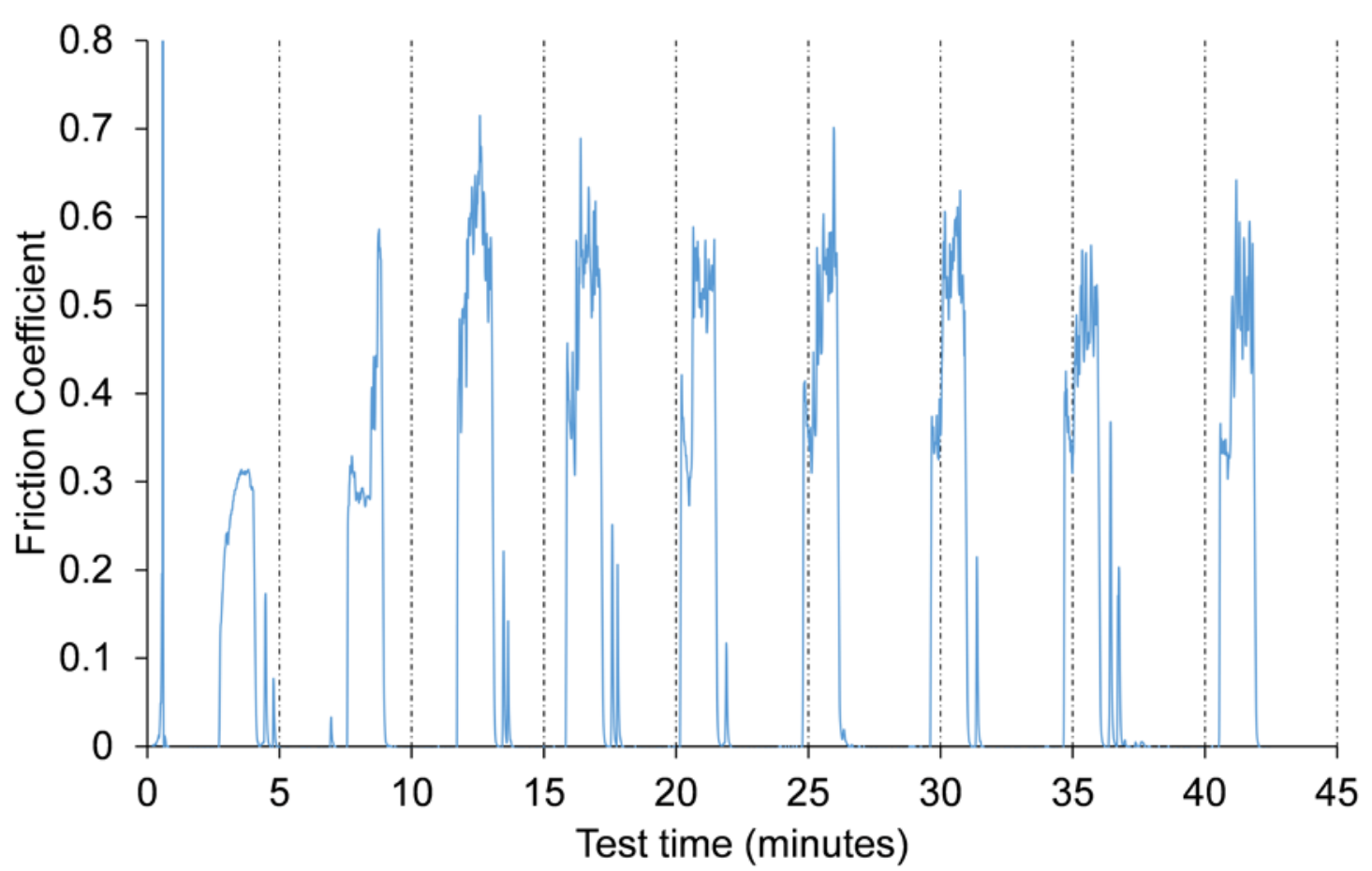

Figure 11: Friction coefficient plotted as a function of test time.

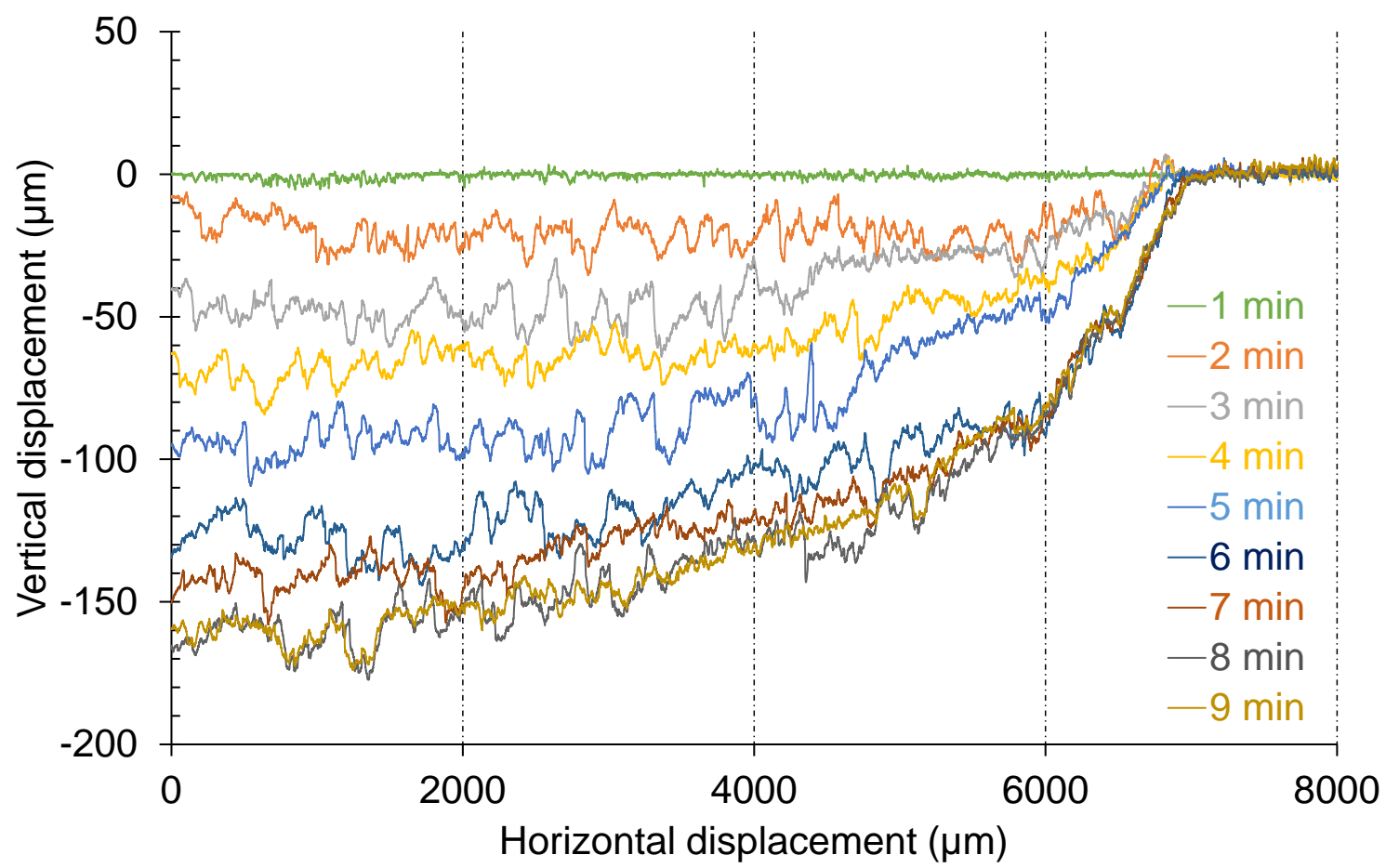

Figure 12: Wear scar profiles measured after every minute of sliding. 


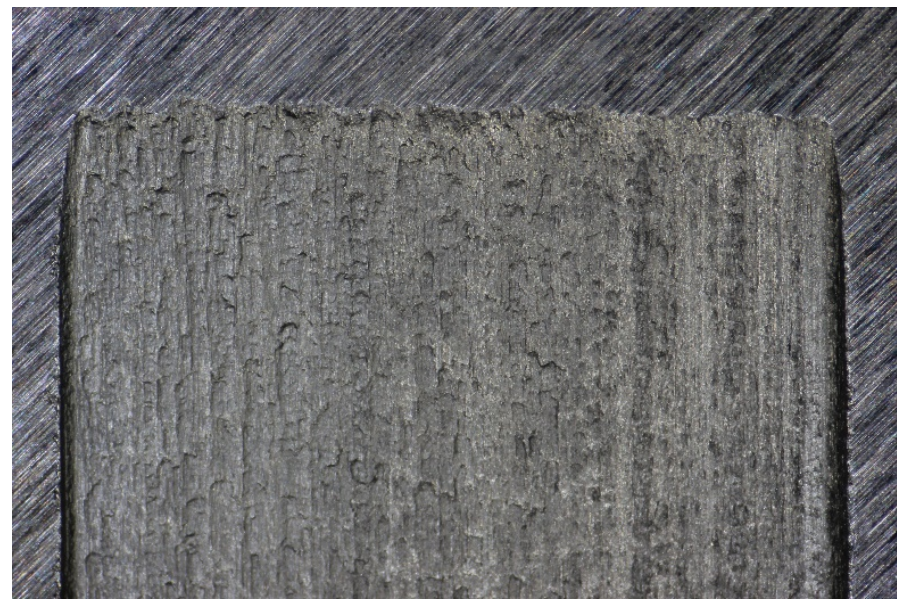

Figure 13: Optical image of the $10 \mathrm{~mm}$ wide cast iron wear scar; the sliding direction was vertical.

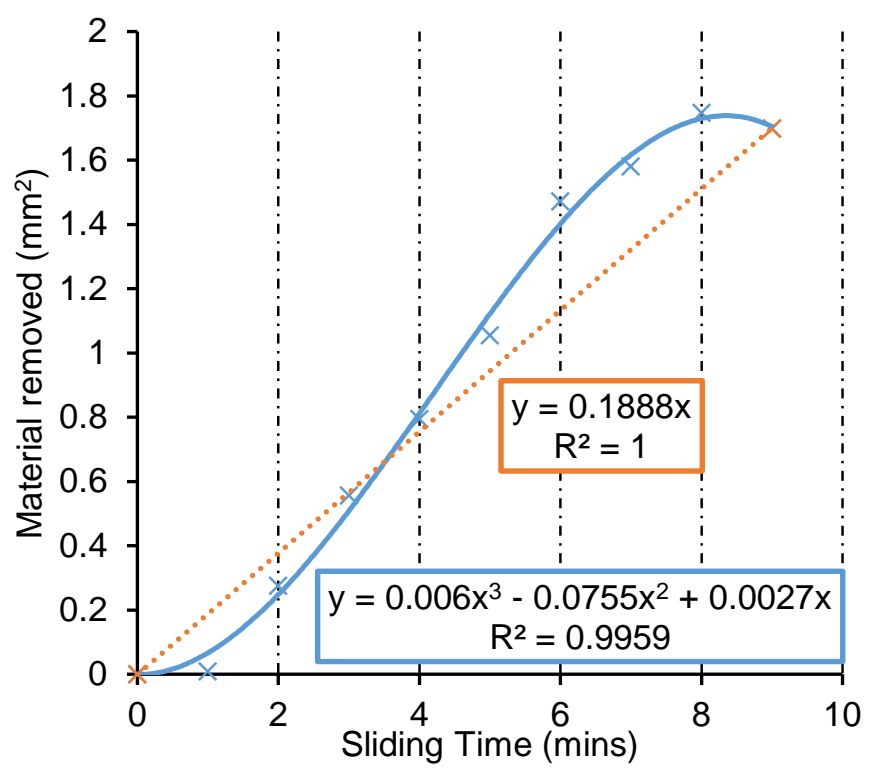

Figure 14: Cumulative wear of the cast iron sample. The in situ profilometer reveals that the wear behaviour is nonlinear and behaves according to the incubation run in steady state model previously discussed.

The friction coefficient and wear of the cast iron sample from the dry sliding wear test are presented in Figures 11 and 12. The friction spikes outside each instance of sliding in Figure 11 were caused by the reciprocating head repositioning at the end of the stroke to expose the wear scar for measurement. The surface profile was measured in the central part of the wear scar, initiating $7 \mathrm{~mm}$ from the stroke reversal position before moving parallel to the direction of sliding towards the unworn ground surface, Figure 13.

The experimental conditions were such that this contact exhibited an incubation period followed by running-in and steady state [13]. The incubation period was accompanied by a friction coefficient of 0.3 and lasted until halfway through the second minute of sliding whereupon the friction coefficient doubled to 0.6 . The decrease in change of wear track depth during the ninth minute of sliding indicated that the running-in regime lasted until the end of the proceeding minute of sliding. This could have been caused by wear debris formed in the unmeasured part of the wear scar being dragged across into the measured part. It is also possible that the change in behaviour was caused by work hardening at the base of the wear track. This transition was proceeded by a gradual decrease in friction coefficient from 0.6 to 0.45 which is consistence with a run-in regime.

The wear data recorded for each minute of sliding highlights the potential danger from recording mass loss at the end of an experiment and extrapolating a linear wear rate, the red line in Figure 14. There were in fact three wear rates, one associated with each wear regime. It is clear that extrapolating the predicted contact lifetime using the annotated wear rate in Figure 14, rather that the steady state, will be inaccurate providing that the contact does not experience another transition. This highlights that the behaviour of a contact is a function of both its past and present conditions.

The cast iron wear scar formed by the end of the dry sliding test was measured by the in situ profilometer and compared with that measured using a Talysurf profilometer equipped with a $2 \mu \mathrm{m}$ standard tip, Figure 15. Due to the difficultly of locating both stylus tips in the same place, the in situ trace was compared against the average of 5 parallel Talysurf profiles. 
The overall shape of both profiles were similar, however the Talysurf profile exhibited a negative skew compared to the unfiltered profile. This was caused by the presence of electrical noise on this unfiltered profile. Comparing the two profiles highlights the effectiveness of the in situ profilometer for accurately measuring wear in situ.

This development of in situ wear monitoring provides information about wear and surface topography evolution pertinent to individual wear regimes that the contact may be operating in at a specific time during an experiment. It has been demonstrated that differences in the running-in damage behaviour can be discriminated using this technique and directly correlated to the observed friction behaviour measured from the tribometer. Integrating both tribometer and profilometer for synchronous operation opens the possibility of automatically measuring differences in surface topography and wear volume arising from pre-determined fluctuations in the instantaneous friction force signal.

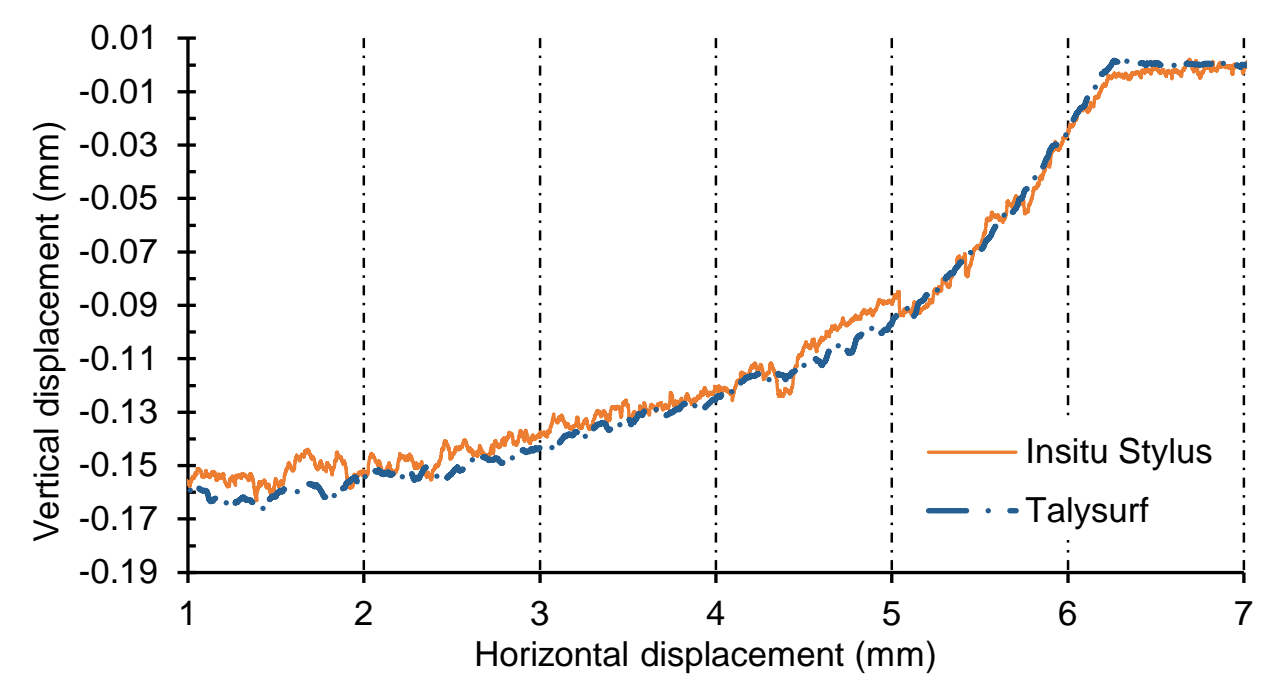

Figure 15: Measurement validation against a Talysurf with a $2 \mu \mathrm{m}$ standard tip.

\section{Conclusions}

- A diamond stylus profilometer based on a Hall effect sensor was manufactured and successfully integrated into a Phoenix Tribology TE77 High Frequency Reciprocating Tribometer.

- The sensor provided satisfactory performance for measuring roughness standards between $\mathrm{R}_{\mathrm{a}} 0.05$ and $1.6 \mu \mathrm{m}$ with an error of less the $0.12 \mu \mathrm{m}$.

- The profilometer was synchronised to measure changes in surface topography from a dry sliding reciprocating contact without the need to disturb the sliding surfaces.

- The in situ profilometer quantified wear regime transitions between incubation, running-in and steady state wear that could be directly correlated to the friction coefficient signal.

- The work opens the possibility of studying surface topography changes that result directly from observable differences in the friction coefficient of a sliding material pair.

\section{Acknowledgements}

The authors would like to acknowledge funding for this project from EPSRC Impact Acceleration Account and the technical expertise provided by Mr Cyrille Favede. All data supporting this study are 
openly available from the University of Southampton repository at http://doi.org/10.5258/SOTON/D0210 


\section{References}

[1] Riahi A R and Alpas A T 2003 Wear map for grey cast iron Wear 255 401-9

[2] Das S, Varalakshmi K, Jayaram V and Biswas S K 2007 Ultra Mild Wear in Lubricated Tribology of an Aluminium Alloy J. Tribol. 129942

[3] P.J. Blau 1981 Mechanism for transitional friction and wear behaviour of sliding metals Wear 72 55-66

[4] Barber G C and Ludema K C 1987 The break-in stage of cylinder-ring wear: a correlation between fired engines and a laboratory simulator Wear 118 57-75

[5] Quinn T F J 1983 Review of oxidational wear - Part I : The orignis of oxidational wear Tribol. Int. 16 257-71

[6] Ludema K C 1984 A review of scuffing and running-in of lubricated surfaces, with asperities and oxides in perspective Wear $100315-31$

[7] Kamps T J, Walker J C, Wood R J, Lee P M and Plint A G 2015 Reproducing automotive engine scuffing using a lubricated reciprocating contact Wear 332-333 1193-9

[8] Kamps T J, Walker J C, Wood R J, Lee P M and Plint A G 2017 Scuf fi ng mechanisms of EN-GJS 400-15 spheroidal graphite cast iron against a 52100 bearing steel in a PAO lubricated reciprocating contact Wear 376-377 1542-51

[9] Aue G K 1975 Piston Ring Scuffing - General Review Piston Ring Scuffing (Mechanical Engineering Publications Ltd) pp 1-5

[10] Blau P J 1991 Running-in: Art or engineering? J. Mater. Eng. 13 47-53

[11] Pawlus P 1997 Change of cylinder surface topography in the initial stage of engine life Wear $20969-83$

[12] Bell J C and Willemse P J 1998 Mid-life scuffing failure in automotive cam-follower contacts Proc. Inst. Mech. Eng. Part J J. Eng. Tribol. 212 259-69

[13] Blau P J 2014 How common is the steady-state? The implications of wear transitions for materials selection and design Wear 332-333 1120-8

[14] Stout K and Blunt L 2000 Development of methods for the characterisation of roughness in three dimensions (London: Penton Press)

[15] ASTM International 2017 ASTM G99 - 17, Standard test method for wear testing with a pinon-disk apparatus (West Conshohocken, PA)

[16] ASTM International 2014 ASTM D3702 - 94(2014), Standard test method for wear rate and coefficient of friction of materials in self-lubricated rubbing contact using a thrust washer testing machine (West Conshohocken, PA)

[17] Stout K J, King T G and Whitehouse D J 1977 Analytical techniques in surface topography and their application to a running-in experiment Wear 43 99-115

[18] Czichos H, Becker S and Lexow J 1989 International multilaboratory sliding wear tests with ceramics and steel Wear 135 171-91

[19] Wang L, Wood R J K, Harvey T J, Morris S, Powrie H E G and Care I 2003 Wear performance of oil lubricated silicon nitride sliding against various bearing steels Wear 255 657-68 
[20] So H 1996 Characteristics of wear results tested by pin-on-disc at moderate to high speeds Tribol. Int. 29 415-23

[21] Dulias U, Fang L and Zum Gahr K H 2002 Effect of surface roughness of self-mated alumina on friction and wear in isooctane-lubricated reciprocating sliding contact Wear 252 351-8

[22] Brunskill H, Harper P and Lewis R 2015 The real-time measurement of wear using ultrasonic reflectometry Wear 332-333 1129-33

[23] Scherge M, Pöhlmann K and Gervé A 2003 Wear measurement using radionuclide-technique (RNT) Wear 254 801-17

[24] Priest M 1996 The wear and lubrication of piston rings (University of Leeds)

[25] Walker J C, Kamps T J and Wood R J K 2012 The influence of start-stop transient velocity on the friction and wear behaviour of a hyper-eutectic Al-Si automotive alloy Wear 306 209-18

[26] Kumar J and Shunmugam M S 2006 A new approach for filtering of surface profiles using morphological operations Int. J. Mach. Tools Manuf. 46 260-70

[27] Raja J, Muralikrishnan B and Fu S 2002 Recent advances in separation of roughness, waviness and form Precis. Eng. 26 222-35

[28] Renegar T B, Soons J, Muralikrishnan B, Villarrubia J S, Zheng A, Vorburger T V and Song J 2012 Stylus Tip-Size Effect on the Calibration of Periodic Roughness Specimens with Rectangular Profiles Icsm3 291-5

[29] Church E L and Takacs P Z 1990 Effects of the non-vanishing tip size in mechanical profile neasuranents 1332 504-14 